\title{
Management Model of Ecological Tourism for the City of Francisco de Orellana
}

\section{Modelo de Gestión de Turismo Ecológico en la Amazonía Ecuatoriana - Orellana}

\author{
R. S. Lara Vásconez ${ }^{1}$, C. A. Hidalgo Mendoza², and D. F. Cedeño Moran ${ }^{3}$ \\ ${ }^{1}$ Escuela Superior Politécnica de Chimborazo, Grupo de Investigación Causana Yachay ESPOCH, \\ Puerto Francisco de Orellana, Ecuador \\ ${ }^{2}$ Escuela Superior Politécnica de Chimborazo, Grupo de Investigación Killari Science ESPOCH, \\ Puerto Francisco de Orellana, Ecuador
}

Memories II International

Congress Forests and

Agroforestry for the 21st

Century

Corresponding Author:

R. S. Lara Vásconez

Published: 21 January 2021

Production and Hosting by

Knowledge E

(c) R. S. Lara Vásconez et al. This article is distributed under the terms of the Creative Commons Attribution License, which permits unrestricted use and redistribution provided that the original author and source are credited.

\section{Abstract}

The purpose of the present study is to design a management model of ecological tourism for the city of Francisco de Orellana. Through the compilation of information and tourist offers, and the analysis of biophysical, sociocultural and economic areas, and plants, superstructures and attractions that appeal to tourists, 4 natural and 5 cultural places were identified. With the application of the environmental impact matrix, it was determined that tourist attractions present a high level of contamination due to the presence of the industrial sector and the inadequate handling of dirty water and solid waste. The most affected tourist attractions are: Napo and Payamino rivers, the Yacu eco-park and the boardwalk; throughout the environmental impact matrix they establish obstacles to environmental remediation. With the results obtained, a sustainable tourism management model was designed based on the objectives of Plan Nacional del Buen Vivir and PLANDETUR 2020. The study concludes that the management model will provide a starting point for the development of sustainable tourism activity in the city.

Keywords: sustainable tourism, management model, environmental sustainability, government policies, environmental impact, strategic plan.

Resumen

El objetivo de la presente investigación es diseñar un modelo de gestión del turismo ecológico para la ciudad de Francisco de Orellana. A través de la recopilación de información, oferta turística y el análisis de áreas biofísicas, socioculturales, económicas, planta turística, superestructura turística y el atractivo turístico donde se identificaron 4 lugares naturales y 5 culturales. Con la aplicación de la matriz de impacto ambiental se determinó que los atractivos turísticos presentan un alto nivel de contaminación debido a la presencia del sector industrial y al manejo inadecuado del agua sucia y los residuos sólidos. Los atractivos turísticos más afectados son: Ríos Napo y Payamino, Payamino El eco parque Yacu y el malecón, a lo largo de la matriz de soluciones, establecen alternativas de remediación ambiental. Con los resultados obtenidos, se diseña un modelo de gestión del turismo sostenible basado en los objetivos del Plan Nacional del Buen Vivir y PLANDETUR 2020. Se concluye que el modelo de gestión proporcionará el comienzo para desarrollar una actividad turística sostenible en la ciudad.

Palabras Clave: turismo sostenible, modelo de gestión, sostenibilidad ambiental, políticas gubernamentales, impacto ambiental, plan estratégico. 


\section{Introducción}

El turismo es una de las actividades económicas más importantes a nivel global, ya que el papel que tiene en la generación de divisas, de empleos y de inversión lo hacen jugar un rol preponderante en las principales economías nacionales, debido a que en los últimos años el turismo en el mundo ha crecido a tasas superiores que el crecimiento de la economía en su conjunto [1], se debe a que las personas que visitan un país no es quien tiene fijada su residencia, por cualquier razón que no sea desempeñar una ocupación remunerada [2]. Francisco de Orellana es uno de los cantones más importantes de la provincia de Orellana, por su actividad petrolera se ha convertido en una de las ciudades más representativas e importantes de la región amazónica y del país. Su auge económico y población surge con el boom petrolero que produjo la migración de otras provincias, actividad que llamó la atención de extranjeros y se establecieron con empresas en la zona. Su territorio es una terraza aluvial conformada y delimitada por los Ríos Coca, Napo y Payamino, los cuales son el marco geográfico e hidrológico de la ciudad, cuenta con servicios básicos, infraestructura hotelera, comercial, financiera y de comunicaciones terrestre, aérea y fluvial; su clima es cálido húmedo, posee una gran biodiversidad, recursos hídricos y manifestaciones culturales [3]. En el año 2013 recibió por parte de empresa CERTIFIKA el sello de calidad turística 'Ecuador, Destino de Vida' y adopto el slogan 'El Coca, La Puerta al Yasuní [4], convirtiéndose en la primera ciudad en recibir este distintivo en el país, mediante el cual se certificó a 42 hoteles y restaurantes del sector, entre sus atractivos más representativos tenemos: Puente río Napo (malecón), parque central, museo arqueológico, eco parque, centros nocturnos de diversión y restaurante que ofrecen comida y bebidas propias de la zona como lo es maito, pincho de mayón y la chicha de chonta. Sin embargo, su gran biodiversidad y recursos hídricos se han visto afectada por la explotación petrolera, fuente principal de la economía de la ciudad. Con la presente investigación se pretende diagnosticar la situación actual de los atractivos turísticos mediante criterios de sostenibilidad y diseñar programas que nos permitan desarrollar el dinamismo turístico en la ciudad de Orellana.

\section{Material y Métodos}

Se elaboró el modelo de gestión de turismo sostenible, donde se establecieron políticas de gestión que contrarrestarán los impactos producidos por las actividades y el manejo de los atractivos, se sustentó a través del modelo realizado por SENPLADES en la Primera Cumbre de turismo sostenible Galápagos 2010 [5]. En el plan nacional del buen vivir 2014-2019: En sus objetivos: 1 'Garantizar una vida digna con iguales oportunidades para todas las personas', 3 'Garantizar los derechos de la naturaleza para las actuales y futuras generaciones', 6 'Impulsar la productividad y competitividad para el crecimiento económico sostenible, de manera redistributiva y solidaria' [6]. En donde la planificación del turismo sostenible responde a las necesidades de los turistas, protege y mejora las oportunidades del futuro. Dentro del plan estratégico Se diseñó y se estableció políticas de gestión de acuerdo a la necesidad que presenta la ciudad de Coca, para 
el desarrollo de la actividad turística con impactos ambientales mínimos, de acuerdo al análisis realizado dentro del taller participativo donde se determinó la situación turística actual de la ciudad. d) Sistemas de manejo de turístico: En base a la metodología del SIMAVIS, se estableció el manejo adecuado de los atractivos turísticos de la ciudad de Coca, 3) Programas del modelo de gestión Para el establecimiento de programas se basó en los objetivos del Plan Nacional del Buen Vivir y áreas funcionales del PLANDETUR 2020, que a través de la matriz lógica se estructuraron los proyectos y actividades a ejecutarse.

\section{Resultados}

Dentro de la urbe de Francisco de Orellana encontramos diversos puntos turísticos y espacios que brindan la posibilidad de crear e implementar nuevos proyectos para potenciar esta actividad económica, sin embargo no se cuenta con herramientas que proporcionen las bases para desarrollar una actividad turística que brinden las garantías de sostenibilidad. El presente modelo pretende convertirse en una guía que ayudará a mejorar el manejo de los atractivos presentes y servirá como base para el desarrollo de nuevos proyectos turísticos en la ciudad a través de la intervención del GAD municipal de Puerto Francisco de Orellana, ente que apoyo el diseño del modelo de gestión de turismo sostenible.

\subsection{Plan estratégico del modelo de gestión}

Misión: El GADM de Francisco de Orellana a través de la subdirección de gestión y desarrollo turístico, impulsará la elaboración y ejecución de proyectos turísticos sostenibles que le permita a la población de Puerto Francisco de Orellana desarrollar y aprovechar el potencial turístico de la ciudad y se posicione como un destino de vida a nivel local, nacional e internacional.

Visión: En 5 años la ciudad de Puerto Francisco de Orellana contará con un sistema turístico habilitado que le permita tener un modelo de gestión de turismo sostenible y garantice un buen manejo turístico, orientado a brindar un servicio de calidad, enmarcado a la concientización de la conservación y respeto hacia los bienes patrimoniales de la ciudad.

Políticas Gobernanza: Se establece políticas y estrategias que permitan una adecuada administración ambiental y turística de la ciudad enfocada en cuatro aspectos importante:

1. Uso óptimo de los recursos naturales

2. Revitalización y fortalecimiento sociocultural de la ciudad

3. Generar actividades económicas viables para la población

4. Protección y conservación ambiental

Descripción: Se pretende alcanzar un desarrollo sostenible a través del establecimiento de políticas y estrategias que respalden la toma de decisiones de cada uno 
de los actores involucrados dentro de la actividad turística, con el objetivo primordial de fortalecer la planificación y gestión de la actividad turística a través de un enfoque coordinado y planificado que nos conllevará a consolidar a la ciudad como un producto turístico y un destino de vida.

El éxito del modelo dependerá del trabajo y gestión en conjunto y participativo entre los entes públicos, prestadores de servicios y personal local de la ciudad, lo que exigirá mejorar su competitividad, planificación, gestión y por ende se fortalecerá la normativa turística y ambiental que rige a la actividad dentro de la ciudad, por lo que se sugiere las siguientes estrategias y políticas:

\section{Estrategias:}

1. Realizar convenios entre el Ministerio de Turismo, Ministerio del ambiente, GADS Municipal y Provincial.

2. Conformar comités en las diversas áreas del turismo, los cuales estarán integrados por los sectores públicos, privados y actores involucrados en la actividad con el objetivo de desarrollar y abarcar todos los sectores turísticos de la ciudad.

3. Crear un organismo de regulación de precios y recategorización en el sector turístico, conformado por el Alcalde, representante de la planta turística, bajo dirección del Ministerio de turismo.

4. Desarrollar herramientas mediante el diseño de un plan maestro de turismo donde se generen los instrumentos necesarios y precisos para lograr el desarrollo de la actividad turística, considerando los criterios de un enfoque integral, interdisciplinario y holístico.

\section{Políticas}

1. Competitividad turística, basada en la calidad de servicios turísticos y la oferta turística de la ciudad.

2. Protección al turística que visita la ciudad, basado en la sanción de una remuneración básica, en reincidencia dos remuneraciones básicas más clausura del lugar por el incumplimiento de las normas regulación de precios establecidos por el organismo de vigilancia turística, por un tiempo de 5 días.

De este Plan Estratégico se generó un concurso: 'MEJORAR LAS FACHADA DE LA PLANTA HOTELERA', con el tema 'Representatividad cultural de la ciudad' del uso óptimo de los recursos naturales y protección ambiental, bajo convenio con el Ministerio del Ambiente y Ministerio de Turismo.

Reingeniería del destino, cuyo objetivo es Diseño y definición del producto turístico sostenible que dé a conocer la biodiversidad natural y patrimonio cultural de la zona.

Posicionamiento del mercado, como objetivo: Posicionar a Puerto Francisco de Orellana como un destino de vida a través la consolidación del sistema turístico de la ciudad.

Descripción: Con la realización de planes de marketing e investigaciones profundos del mercado se pretende proporcionar las herramientas necesarias para la creación 
de futuros productos turísticos innovadores y brindar a los turistas un servicio con altos estándares de calidad, tomando como la principal meta incrementar la demanda turística de la ciudad.

Paralelo a los antes mencionado se deberá trabajar concientizando a la población local y actores involucrados mediante la elaboración de cronogramas anuales de capacitación con temas referentes a la generación de un turismo sostenible con la finalidad de fortalecer el sistema turístico de la ciudad y así convertirla en un destino de vida capaz de satisfacer todas le necesidades de los turistas.

\section{Simatur:}

Objetivo: Proporcionar una herramienta metodológica a los actores que se encuentran involucrados para que puedan brindar un servicio de calidad con el menor impacto ambiental posible.

Descripción: EI SIMAVIS está pensado para el manejo de los visitantes dentro de las áreas protegidas, se puede llegar adaptar en diversos espacios en donde se desarrolle la actividad turística gracias a su criterio de calidad de la experiencia de visitantes y al respeto que se debe de tener a los objetivo de conservación expuestos en el la Constitución de la República del Ecuador, Plan de Buen Vivir y PLANDETUR, se caracteriza por tomar como referencia los aspectos naturales, físico- geográfico, sociales, culturales e intervención humana.

Con la adaptación de esta metodología al SIMATUR se pretende alcanzar los mismos objetivos, pero enfocados a atractivos turísticos dentro de la zona urbana, para lo cual se tomará los siguientes componentes:

\section{Decisiones de manejo}

\section{Zonificación}

Uno de los principales objetivo de la metodología es la conservación del patrimonio natural y cultural en cada uno de los atractivos turístico con los que cuenta el casco urbano destinado para uso público, para lo cual basamos el SIMATUR en los principio de la zonificación expuesto por el [7], lo que tendremos:

Compatibilizar los objetivos de conservación de un área con el uso turístico susceptible en los mismos.

Desarrollar prescripciones respecto de las intervenciones y manejo físico, social y administrativo deseado. Como por ejemplo podemos citar al parque central de la ciudad de Francisco de Orellana con la siguiente zonificación estructural:

1. Área de juegos infantiles

2. Alimentos y Bebidas

3. Área de descanso

4. Plaza de eventos

5. Senderos

6. Servicios higiénicos 
Cálculo de la capacidad de carga: Cada atractivo deberá establecer y exponer la capacidad de carga del sitio través de la aplicación de diversas fórmulas que permitan realizar este cálculo, dentro del [7] elnúmero de visitantes es un asunto de calidad de visitas. La meta general es tener la menor cantidad de visitantes posible en un sitio al mismo tiempo, en especial en aquellos sitios en los que los visitantes, van a sentir una mayor satisfacción y su experiencia será mejor cuando el entorno ofrece un cierto nivel de exclusividad (pocos encuentros con otros visitantes, número reducido de grupos y pasajeros).

La capacidad de carga diaria aplicada de manera exclusiva no es relevante. Diez grupos en un sitio al mismo tiempo pueden causar gran contaminación visual y eventualmente impactos sobre la naturaleza o el atractivo, si a esto se suma el no cumplimiento de las reglas de uso y comportamiento los resultados serán peores.

Para calcular la capacidad de carga de los diferentes atractivos turístico de la ciudad se sugiere la metodología por [8], tomando en cuenta lo diversos indicadores que lo conforman como son ecológicos, físicos, económicos, sociales y culturales.

\subsection{Programas del modelo de gestión de turismo sostenible}

Los programas del modelo de gestión de turismo sostenible se los realizó tomando como referencia los objetivos del plan nacional de buen vivir y las áreas funcionales del PLANDETUR 2020.

\section{Discusión}

Según el modelo de Sainaghi [9]. Se expresa que lo mas importante con respecto al manejo de los destinos es saber ¿Qué hacer? y ¿Cómo hacerlo?, en su metodología enfoca principalmente la gestión estratégica, la dinamica y la estatica [10]. Para estos autores el punto clave es la la demanda, tiene que ver con el análisis de las Corrientes turísticas de manera estratégica en un ambito regional y que se pueda controlar, concluyendo que la demanda es la que activa la oferta y no al revés. En cuanto a modelos funcionales el ejemplo que presenta el distrito metropolitano de Caracas, Ornés Vásquez [11] ofrece una diferente percepción, con respecto a relaciones diversas y funciones, con cuatro componentes que estructuran el modelo, (planificación; inversiones y proyectos; mercadeo; y desarrollo social y económico) y en base a estos parametros se etableceran las lineas estratégicas de acción' [11] (p. 103). Segun [12] la naturaleza dinámica de los destinos se ve reflejada los modelos de proceso, a través de los mismos se visualiza la naturaleza de la dinamica de gestión de los destinos. De acuerdo con Vera et al. [13] y González Cornejo y Rivas Ortega [14], No es suficiente contar con un modelo que contenga una estrategia, con objetivos, actividades y una correcta organización, lo que realmente se necesita es una evaluación constante y continua de los modelos, que pruebe que las acciones se siguen cumpliendo de forma continua en la práctica [15]. Más bien, es una actividad dinámica y constante y los modelos deberían demostrar esta continuidad de acciones. 
Table 1

Matriz lógica.

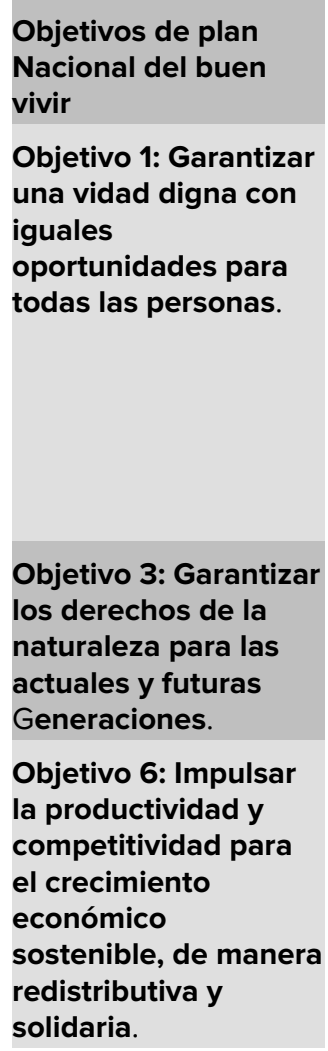

Plandetur de funcionales

Administrar en el sistema turístico. Educación y capacitación de recursos humanos.

Medidas para la gestión ambiental para el desarrollo el turismo sotenible.

\section{Desarrollo del destino y} facilidades turísticas.

. producto y posicionamiento del mercado.

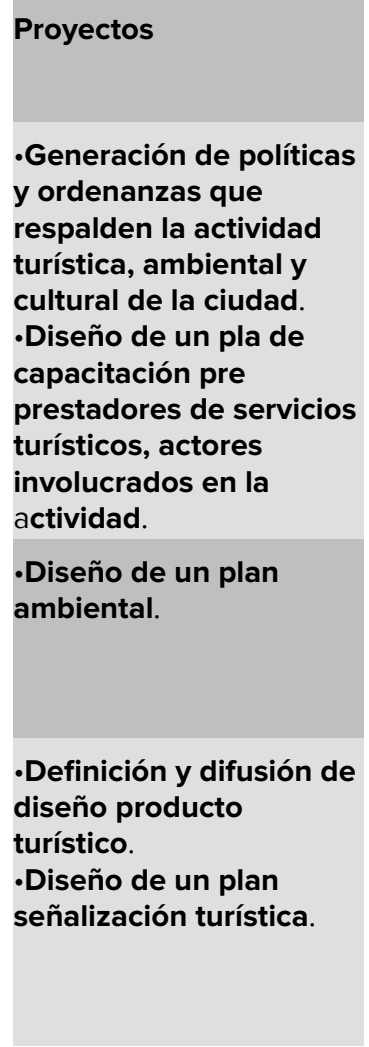

Proyectos

- Generación de políticas y ordenanzas que respalden la actividad turística, ambiental y cultural de la ciudad. - Diseño de un pla de citación pre turísticos, actores involucrados en la

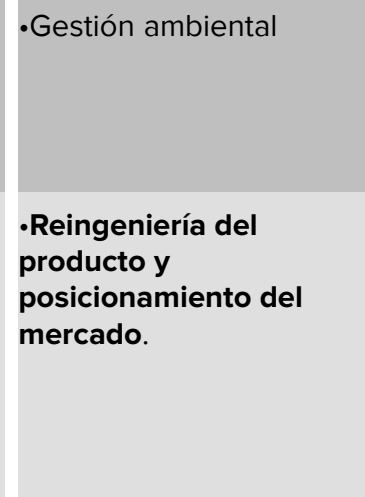

Resumen de Programas con proyectos implementados siguiendo los objetivos del Plan Nacional del Buen Vivir.

EI GAD Municipal de Francisco de Orellana no cuenta con un módelo de gestión en donde se establezca las necesidades reales de la ciudad, que permita cambiar aquella visión y aquel pensar que el único potencial es el petróleo y que todo gira alrededor de aquel sector, en base a lo expuesto se ha visto necesario desarrollar un modelo de manejo turístico y ambiental con la finalidad de generar estrategias que permitan alcanzar un desarrollo sostenible, mediante el cual se logre un equilibrio entre la conservación y preservación de los recursos naturales con las actividades turísticas desarrolladas, para lo cual se a estudiado algunos modelos de gestión de destinos, pero para comprovar su eficacia en cuanto a la aplicación en el campo práctico se sugiere utilizaar este modelo en una prueba empirica, para luego evaluar sus verdadero alcance en base a los resultados que se obtengan en el mediano plazo.

\section{Conclusiones}

En base a bibliografía consultada se diseñaron cuatro programas basado en las necesidades de la ciudad que se realizarán en un tiempo determinado de cinco años, lo que convierte al modelo de gestión en un proyecto de corto plazo. 
El modelo de gestión dará las pautas para desarrollar una actividad turística sostenible atractiva para el turista y amigable con el ambiente, sus postulados se basan en el buen vivir y en la constitución de la República del Ecuador cuyo eslogan gira alrededor de la PACHA MAMA.

\section{Agradecimiento}

Agradecemos a los colegas que colaboraron en el diseño y redacción del presente artículo.

\section{Conflicto de Interés}

No existe un interés particular por parte de los autores ni de la entidad científica que pueda afectar directa o indirectamente a los resultados.

\section{References}

[1] Meixueiro G. (Julio de 2008). Impacto de la actividad turística en el desarrollo local [Impact of tourism activity on local development]. Disponible en: http://www3.diputados.gob.mx/.../limpacto_ \{\%\}20turismo_desarrollo_docto48.pdf. Spanish

[2] Di-Bella MG. Introducción al Turismo [Introduction to tourism]. México: Trillas S.A; 1991. Spanish

[3] Orellana GM. Plan de Desarrollo y Ordenamiento Territorial Municipal de Francisco de Orellana. Orellana: s/n. 2014-2019. Spanish

[4] Turismo M. Ministerio de Turismo [Internet]. 17 de 10 de 2013 [Recuperado el 05 de 10 de 2019]. De Noticias: https://www.turismo.gob.ec/el-coca-es-el-primer-destino-de-vida-de-ecuador/. Spanish

[5] Quirola D. Análisis del concepto de buen vivir aplicado a la planificación del turismo sostenible [Internet]. Primera Cumbre de Turismo Sostenible Galápagos. 2010. Disponible en: http://www.optur.org/docs/ SENPLADES\{\%\}20Dania\{\%\}20Quirola\{\%\}20Turismo\{\%\}2 0Sostenible\%20230910.ppsx. Spanish

[6] Senplades. Plan Nacional de Desarrollo. Quito - Ecuador: CNP. 2017-2021. Spanish

[7] SIMAVIS (2008)

[8] Cifuentes (1992)

[9] Sainaghi (2006).

[10] Beritelli P, Bieger T, Reinhold S, Laesser C. “The St. Gallen model for destination management”. 2015.

[11] Ornés Vásquez S.“La gestión pública del turismo en el distrito metropolitano de Caracas: fortalezas, debilidades y nuevos desafíos”. GestiónTurística. 2009; 12: 85-108.

[12] Pearce DG."Destinos turísticos: conceptos e implicaciones para su gestión en tiempos de cambio”. En: Cànoves G, López Palomeque F. (Eds.) Turismo y territorio: innovación, renovación y desafíos. Valencia: Tirant Humanidades. 2014. p. 21-34. Spanish

[13] Vera, et al. (2011).

[14] González Cornejo \& Rivas Ortega (2008).

[15] Pearce DG. "Plans and practitioners' perspectives in New Zealand”. Tourism Planning \& Development. 2015

[16] SERNATUR. Modelo de gestión integral de destinos turísticos [Internet]. 2008. Disponible en: http://www.sernatur.cl/wp-content/plugins/download-monitor/download.php?id= Modelo-Gesti\{\%\}C3\{\%\}B3n-Integral-Mayo2008.pdf. Spanish 